M. Nisio

Nagoya Math. J.

Vol. 123 (1991), 13-37

\title{
OPTIMAL CONTROL FOR STOCHASTIC PARTIAL DIFFERENTIAL EQUATIONS AND VISCOSITY SOLUTIONS OF BELLMAN EQUATIONS
}

\author{
MAKIKO NISIO
}

\section{§1. Introduction}

Recently M. G. Crandall and P.L. Lions developed the viscosity theory on nonlinear equations in infinite dimensions and optimal control in Hilbert spaces, in two series of papers, [1], [4].

In this article we will study of optimal control of stochastic partial differential equations and viscosity solutions of Bellman equation (1.1) below,

(1.1) $\sup _{u}\left(-\frac{1}{2}\left(D^{2} \nu(\phi) M \phi, M \phi\right)-(D \nu(\phi), L(u) \phi)+\lambda \nu(\phi)-F(\phi)\right)=0$,

where $D$ and $D^{2}$ denote the first and second Fréchet differentials and $M$ and $L(u)$ are the first and second order differential operators respectively (see (2.1)).

Let us consider the following stochastic partial differential equation, (SPDE in short)

$$
\begin{aligned}
d q(t)= & \sum_{i j=0}^{d} \frac{\partial}{\partial x_{i}}\left(a^{i j}(x, U(t)) \frac{\partial}{\partial x_{j}} q(t, x)+f^{i}(x, U(t))\right) d t \\
& +\sum_{k=1}^{m}\left(\sum_{i=0}^{d} b_{k}^{i}(x) \frac{\partial}{\partial x_{i}} q(t, x)+g_{k}(x)\right) d W^{k}(t),
\end{aligned}
$$

where $W=\left(W^{1}, \cdots, W^{m}\right)$ is an $m$-dimensional standard Wiener process and $U(t)$ an admissible control. We will define the criterion $J$ by

$$
J(\phi, U)=E \int_{0}^{\infty} e^{-\lambda t} F(q(t, \phi, U)) d t
$$

where $q(t, \phi, U)$ denotes a solution of (1.2) starting at $\phi$. The function $V$ defined by

Received March 15, 1990. 


$$
V(\phi)=\inf _{U} J(\phi, U)
$$

is called the value function. The SPDE (1.1) describes intuitively a physical object governed by a partial differential equation with random perturbation, which has been investigated from various view points, (see references in [5]). But other important example is the Zakai equation for controlled partially observed diffusions. In this case, inhomogeneous term $f^{i}$ and $g_{k}$ are zero and $b_{k}^{i}$ arises from the correlation between system and observation noises.

We mainly study regularity of the value function, assuming ellipticity codition (see (A.2)) and smoothness of coefficients. Since the value function becomes semi-concave, we can show its differentiability via convex analysis arguments, (Theorem 4.2). Appealing to this property, we demonstrate the relationship between a viscosity solution of (1.1) and the value function.

We will devote Section 2 to the study of optimal control for (1.2), according to [5]. We introduce a relaxed system and show the existence of an optimal relaxed system, (Theorem 2.1). Section 3 deals with smooth properties of the criterion $J(\phi, U)$ defined by (1.3) and the value function (1.4). From the regularity assumptions on coefficients, we can easily see the differentiability of the criterion, (Proposition 3.1 ) and semi-concaveness of the value function, (Proposition 3.2). Using these properties, we discuss directional derivatives, via convex analysis, and prove that the value function is densely Gâteux differentiable in Section 4.

Section 5 is devoted to the study of a connection between the value function and the Bellman equation (1.1). We introduce a subsolution (in viscosity sense) according to [4], but we require one condition which says that its super differentials are densely non-empty, (see (5.16)). The value function turns out to be a subsolution of (1.1), (Theorem 5.1) and the maximum subsolution, which satisfies sub-Bellman principle, (Theorem 5.2). When $b_{k}^{i}$ and $g_{k}$ in (1.2) are zero, the SPDE (1.2) can be regarded as a parabolic equation and control problems turns out to be deterministic ones. Section 6 is a supplement and deals with a deterministic control.

The author wishes to express her sincere thanks to the referee for his valuable suggestions.

\section{§ 2. Optimal control for SPDE}

Let $\Gamma$ be a convex compact metric space. We call it a control region. 
Let us define the operators $L(u)$ and $M=\left(M_{1}, \cdots, M_{m}\right)$ by

$$
\begin{array}{r}
L(u) \psi(x)=\sum_{i j=0}^{d} \frac{\partial}{\partial x_{i}}\left(a^{i j}(x, u) \frac{\partial}{\partial x_{j}} \psi(x)+f^{i}(x, u)\right), \\
\text { for } \quad x \in \mathbf{R}^{d}, \quad u \in \Gamma,
\end{array}
$$

and

$$
M_{k} \psi(x)=\sum_{i=0}^{d} b_{k}^{i}(x) \frac{\partial}{\partial x_{i}} \psi(x)+g_{k}(x), \quad \text { for } x \in \mathbf{R}^{d},
$$

respectively, where $\partial / \partial x_{0}=$ identity, and $a^{i j}, f^{i}, b_{k}^{i}$ and $g_{k}$ are bounded and uniformly continuous.

We denote by $H^{k}$ the Sobolev space of real valued functions defined on $\mathbf{R}^{d}$, with the following norm,

$$
\|\psi\|_{k}^{2}=\sum_{|\alpha| \leq k}\left\|D^{\alpha} \psi\right\|^{2}
$$

where \|\|$=L^{2}\left(R^{d}\right)$-norm, $\alpha=\left(\alpha^{1}, \cdots, \alpha^{d}\right)=$ multi-index with non-negative integer $\alpha^{i},|\alpha|=\alpha^{1}+\cdots+\alpha^{d}$ and $D^{\alpha}=\left(\partial / \partial x_{1}\right)^{\alpha 1} \cdots\left(\partial / \partial x_{d}\right)^{\alpha^{d}}$. $\mathcal{S}$ denotes the subspace of $H^{4}$ with the norm defined by

$$
\|\psi\|\|=\| \psi\left\|_{4}^{2}+\right\|\left(1+|x|^{2}\right) \psi \|_{1}^{2} .
$$

For simplicity, we put $H^{0}=H\left(=L^{2}\left(\mathbf{R}^{d}\right)\right)$, if no confusion occurs.

Now we introduce the following conditions.

(A.1) $\quad D^{\alpha} a^{i j}, D^{\alpha} b_{k}^{i}(0 \leq|\alpha| \leq 4, i, j=0,1, \cdots d, k=1,2, \cdots, m)$ are bounded and uniformly continuous.

(A.2) ellipticity condition. $a^{i j}=a^{j i}, i, j=1, \cdots d$ and $\left(a^{i j}-(3 / 2) b^{i} \cdot b^{j}\right)_{i, j=1, \cdots, d}$ is a non-negative definite matrix, where $b^{i}=\left(b_{1}^{i}, \cdots, b_{m}^{i}\right)$ and "." means the inner product in $\mathbf{R}^{m}$.

(A.3) $f^{i}(\cdot, u), g_{k} \in \mathfrak{S}(\mathrm{i}=0, \cdots, d, k=1, \cdots, m)$ and their $\mathfrak{S}$ norms are bounded in $u$.

Hereafter we always assume (A.1)-(A.3) and for simplicity, we say

$$
\left|D^{\alpha} a^{i j}(x, u)\right| \leqq K, \quad\left|D^{\alpha} b_{k}^{i}(x)\right| \leqq K, \quad\left\|\left|f ^ { i } ( \cdot , u ) \left\|\left|\leqq K, \quad\left\|\left|g_{k}\right|\right\| \leqq K .\right.\right.\right.\right.
$$

To study relaxed systems (in wider sense), we need the following spaces. By $\Lambda$ we denote the set of all Borel measures on $[0, \infty) \times \Gamma$, such that

$$
\lambda([0, s] \times \Gamma)=s, \quad \text { for any } s \geqq 0 .
$$

Endowing with the vague topology, we have the following proposition, 
Proposition 2.1. $\Lambda$ is a compact metric space.

Proof. Let us see $A_{n}=\{\lambda /[0, n] \times \Gamma ; \lambda \in \Lambda\}$, where $\lambda / X$ is the restriction of $\lambda$ on $X$. The induced topology on $\Lambda_{n}$ turns out to be the weak convergence topology and $\Lambda_{n}$ becomes a compact metric space by applying the Prohorov metric, say $\rho_{n}$. Since $\Lambda$ is metrized by $\rho=\sum 2^{-n} \min \left(1, \rho_{n}\right)$, we complete the proof.

Let us set $B(\Gamma)=$ Borel field on $\Gamma, \sigma_{t}(\Lambda)=$ the $\sigma$-field generated by $\{\lambda([0, s] \times A) ; s \leqq t, A \in B(\Gamma)\}$ and $\sigma(\Lambda)=$ the $\sigma$-field generated by $\sigma_{t}(\Lambda)$, $t>0$. Let $\mathbf{P}=\mathbf{P}(\Lambda)$ be the space of all probabilities on $(\Lambda, \sigma(\Lambda))$, endowed with the weak convergence topology. Then Prohorov's theorem asserts

Proposition 2.2. $\mathbf{P}$ is a compact metric space.

By virtue of (2.4), $\lambda$ has a $\sigma_{t}(\Lambda)$-adapted kernel $\lambda^{\prime}$, namely $\lambda(d t, d u)=$ $\lambda^{\prime}(t, d u) d t$, and $\lambda^{\prime}(t, d u)$ is a probability on $\Gamma$ for almost all $t$. Moreover, if $\hat{\lambda}$ is a kernel of $\lambda$, then $\hat{\lambda}(t, \cdot)=\lambda^{\prime}(t, \cdot)$ for almost all $t$. Let us set

and

$$
\tilde{h}(t, x, \lambda)=\int_{\Gamma} h(x, u) \lambda^{\prime}(t, d u), \quad \text { for } h=a^{i j} \text { and } f^{i},
$$

$$
\begin{aligned}
\tilde{L}(t, \lambda) \psi(x) d t & =\int_{\Gamma} L(u) \psi(x) \lambda^{\prime}(t, d u) d t \\
& =\sum_{i j=0}^{d} \frac{\partial}{\partial x_{i}}\left(\tilde{a}^{i j}(t, x, \lambda) \frac{\partial}{\partial x_{i}} \psi(x)+\tilde{f}^{i}(t, x, \lambda)\right) d t .
\end{aligned}
$$

Now we introduce a relaxed system.

Definition 2.1. $\mathscr{R}=\left(\Omega, \mathscr{F}, \mathscr{F}_{t}, \mathscr{P}, W, \mu\right)$ is called a relaxed system, if

$$
\left(\Omega, \mathscr{F}, \mathscr{F}_{t}, \mathscr{P}\right) \text { is a probability space with filtration } \mathscr{F}_{t}
$$

$$
W \text { is an } \mathscr{F}_{t} \text {-adapted } m \text {-dimensional Wiener process with } W(0)=0
$$

and

(2.8) $\mu$ is an $\mathscr{F}_{t}$-adapted $\Lambda$-valued random variable ( $\Lambda$-r.v. in short).

Namely $\mu(A \times B)$ is $\mathscr{F}_{t}$-measurable, whenever $A \in B[0, t]$ and $B \in B(\Gamma)$.

For simplicity, we put $\mathscr{R}=(W, \mu)$, if no confusion occurs, and sometimes we call $\mu$ a relaxed control. $\mathfrak{R}$ denotes the totality of relaxed systems. Let $\pi(\mathscr{R})$ be the image measure on $C\left(\left([0, \infty) ; \mathbf{R}^{d}\right) \times \Lambda\right.$ by $\mathscr{R}=$ $(W, \mu)$. Again endowing the weak convergence topology on the space $\Pi=\{\pi(\mathscr{R}) ; \mathscr{R} \in \mathfrak{R}\}$, we have the following proposition. 
Proposition 2.3. II is a compact metric space.

Proof. Since $W$ is a Wiener process and $\Lambda$ is compact, $\Pi$ is tight by the weak convergence topology. Put $\Omega=C\left([0, \infty) ; \mathbf{R}^{d}\right) \times \Lambda$ and $(w, \lambda) \in \Omega$. We define the usual $\sigma$-field and $\mathscr{F}_{t}$,

$$
\mathscr{F}=\sigma\{w(s), s \in[0, \infty)\} \times \sigma(\Lambda) \text { and } \mathscr{F}_{t}=\sigma\{w(s), s \leqq t\} \times \sigma_{t}(\Lambda) .
$$

Suppose $\pi_{n}(\in \Pi)$ converges to $\pi$ weakly. Then $\pi(\cdot \times \Lambda)$ is a Wiener measure. Moreover, for any bounded continuous $f$ and $g$, we see

$$
\begin{gathered}
\int_{\Omega} f\left(w\left(t_{1}+t\right)-w(t), \cdots, w\left(t_{k}+t\right)-w(t)\right) g\left(w\left(s_{1}\right),\right. \\
\left.\cdots, w\left(s_{j}\right), \mu\left(A_{1} \times B_{1}\right), \cdots, \mu\left(A_{j} \times B_{j}\right)\right) d \pi_{n} \\
=\int_{\Omega} f\left(w\left(t_{1}\right), \cdots, w\left(t_{k}\right)\right) d \pi_{n} \int_{\Omega} g\left(w\left(s_{1}\right), \cdots, w\left(s_{j}\right), \mu\left(A_{1} \times B_{1}\right),\right. \\
\left.\cdots, \mu\left(A_{j} \times B_{j}\right)\right) d \pi_{n}
\end{gathered}
$$

whenever $s_{1}, \cdots, s_{j} \leqq t$ and $A_{1}, \cdots, A_{j} \subset[0, t]$. As $n \rightarrow \infty$, we have the same equality replacing $\pi_{n}$ by $\pi$. Now setting $W(w, \lambda)=w$ and $\mu(w, \lambda)=\lambda, W$ becomes an $\mathscr{F}_{t}$-Wiener process and $\pi \in \Pi$. This completes the proof.

Definition 2.2. We say $\mathscr{R}_{n}$ converges to $\mathscr{R}$ (put $\mathscr{R}_{n} \rightarrow \mathscr{R}$ ), if $\pi\left(\mathscr{R}_{n}\right.$ ) $\rightarrow \pi(\mathscr{R})$ weakly.

Consider the $\operatorname{SPDE}(2.9)$ for $\mathscr{R}=(W, \mu)$,

$$
d q(t)=\tilde{L}(t, \mu) q(t) d t+M q(t) d W(t), \quad q(0)=\phi(\in \mathfrak{S}) .
$$

An $H^{1}$-valued $\mathscr{F}_{t}$-adapted process $q=q(\cdot, \phi, \mathscr{R})$ is called a solution of (2.9), if (2.10) and (2.11) hold.

$$
E \int_{0}^{T}\|q(t)\|_{1}^{2} d t<\infty, \quad \text { for any } T<\infty,
$$

and for any $\eta \in C_{0}^{\infty}$ (smooth function on $\mathbf{R}^{d}$ with compact support) and almost all $t$

$$
\begin{aligned}
(q(t), \eta)=(\phi, \eta) & +\int_{0}^{t}\langle\tilde{L}(s, \mu) q(s), \quad \eta>d s \\
& +\int_{0}^{t}(M q(s), \eta) d W(s), \quad \text { with probability } 1,
\end{aligned}
$$

holds, where $()=,L^{2}\left(\mathbf{R}^{d}\right)$-inner product and $\langle\rangle=$, duality pairing between $H^{-1}$ and $H^{1}$ under $H^{0}=\left(H^{0}\right)^{*}$ (=dual space of $H^{0}$ ). Clearly (2.11) does not depend on any special choice of kernel $\mu^{\prime}$. 
According to Krylov-Rozovskii [3] (cf. [5]), there is a unique solution $q=q(\cdot, \phi, \mathscr{R})$ in the function space below

(2.12) $q \in L^{2}((0, T) \times \Omega ; \mathfrak{S}) \cap L^{2}\left(\Omega ; C\left(0, T ; H^{3}\right)\right), \quad$ for $T<\infty$.

Moreover, there are two constants $N=N(T)$ and $c$, independent of $\mathscr{R}$, such that

$$
\begin{aligned}
& E\left(\sup _{t \leqq T}\|q(t)\|_{k}^{2}\right) \leqq N\left(\|\phi\|_{k}^{2}+A_{k}\right), \quad \text { for } k=0,1,2,3, \\
& E\left(\sup _{t \leqq T}\|q(t)\|_{k}^{4}\right) \leqq N\left(\|\phi\|_{k}^{4}+B_{k}\right), \quad \text { for } k=0,1,2,3, \\
& E\|q(t)\|^{2} \leqq e^{c t}\left(\|\phi\|^{2}+A_{0}\right), \quad \text { for } t>0
\end{aligned}
$$

and

$$
\begin{aligned}
E\left(\sup _{s \leqq t \leqq}\|q(t)-q(s)\|_{j}^{2}\right) \leqq N(\theta-s)\left(\|\psi\|_{j+1}^{2}+A_{j+1}\right) & \\
& \text { for } \theta \leqq T \text { and } j=0,1,2
\end{aligned}
$$

where

$$
A_{k}=\sup _{u} \sum_{i=0}^{d}\left\|\frac{\partial}{\partial x_{i}} f^{i}(\cdot, u)\right\|_{k}^{2}+\sum_{j=1}^{m}\left\|g_{j}\right\|_{k+1}^{2}
$$

and

$$
B_{k}=\sup _{u} \sum_{i=0}^{d}\left\|\frac{\partial}{\partial x_{i}} f^{i}(\cdot, u)\right\|_{k}^{4}+\sum_{j=0}^{m}\left\|g_{j}\right\|_{k+1}^{4} \cdot
$$

Now we recall how $q$ depends on $\mathscr{R}$ and $\phi$.

Proposition 2.4 ([5]). Support $\mathscr{R}_{n} \rightarrow \mathscr{R}$. Then, for $\phi \in \mathfrak{K}$

$$
q\left(t, \phi, \mathscr{R}_{n}\right) \rightarrow q(t, \phi, \mathscr{R}) \quad \text { in law as } H^{1} \text {-r.v. }
$$

For $\phi, \psi \in \mathfrak{S}$, we see

(2.18) $\quad E\left(\sup _{t \leqq T}\|q(t, \phi, \mathscr{R})-q(t, \psi, \mathscr{R})\|_{k}^{2} \leqq N\|\phi-\psi\|_{k}^{2}\right.$, for $k=0,1,2,3$,

and $p(t, \phi-\psi, \mathscr{R})=q(t, \phi, \mathscr{R})-q(t, \psi, \mathscr{R})$ satisfies the linear SPDE (2.19) below

$$
\left\{\begin{aligned}
d p(t, x)= & \sum_{i, j=0}^{d} \frac{\partial}{\partial x_{i}}\left(\tilde{a}^{i j}(t, x, \mu) \frac{\partial}{\partial x_{j}} p(t, x)\right) d t \\
& \quad+\sum_{k=1}^{m} \sum_{i=0}^{d} b_{k}^{i}(x) \frac{\partial}{\partial x_{i}} p(t, x) d W^{k}(t) \\
p(0, x)= & \phi(x)-\psi(x) .
\end{aligned}\right.
$$


Let $F: H \rightarrow \mathbf{R}^{1}$ be bounded and Lipschitz continuous, say

$$
|F(\phi)| \leqq b, \quad \text { for } \phi \in H
$$

and

$$
|F(\phi)-F(\psi)| \leqq a\|\phi-\psi\|
$$

For $\mathscr{R} \in \Re$, we will define the cirterion $J$ and the value function $V$ as follows

$$
J(\phi, \mathscr{R})=E \int_{0}^{\infty} e^{-\lambda t} F(q(t, \phi, \mathscr{R})) d t
$$

where $\lambda(>0)$ is a given constant and

$$
V(\phi)=\inf _{\mathscr{R}} J(\phi, \mathscr{R}),
$$

respectively. Since $J(\phi, \mathscr{R})$ is continuous in $\mathscr{R}$ by Proposition 2.4, Proposition 2.3 asserts the following theorem.

THEOREm 2.1. There is an optimal relaxed system $\mathscr{R}^{*}=\mathscr{R}^{*}(\phi)$, namely

$$
V(\phi)=J\left(\phi, \mathscr{R}^{*}\right) .
$$

From (2.18) and (2.21), we can easily see that, for $\varepsilon>0$, there is $\delta=\delta(\varepsilon)>0$ such that

$$
|J(\phi, \mathscr{R})-J(\psi, \mathscr{R})|<\varepsilon, \quad \text { for any } \mathscr{R} \in \Re,
$$

whenever $\phi, \psi \in \mathfrak{F}$ and $\|\phi-\psi\|<\delta$. Therefore

$$
|V(\phi)-V(\psi)|<\varepsilon, \quad \text { for } \phi, \psi \in \mathfrak{S} \text { and }\|\phi-\psi \cdot\|<\delta .
$$

Since $\mathfrak{S}$ is dense in $H, J(\cdot, \mathscr{R})$ and $V$ can be extended on $H$, say $\bar{J}$ and $\bar{V}$. Moreover $\bar{J}$ and $\bar{V}$ satisfy (2.25) and (2.26) respectively, and

$$
\bar{V}(\phi)=\inf _{\mathscr{R}} \bar{J}(\phi, \mathscr{R}), \quad \text { for } \phi \in H
$$

still holds. $\bar{J}(\phi, \mathscr{R})$ is also continuous in $\mathscr{R}$, by (2.25). So Proposition 2.3 derives

Proposition 2.5. There is $\overline{\mathscr{R}}=\overline{\mathscr{R}}(\phi), \phi \in H$, such that

$$
\bar{V}(\phi)=\bar{J}(\phi, \overline{\mathscr{R}}) \text {. }
$$

When a relaxed system $\mathscr{R}=\left(\Omega, \mathscr{F}, \mathscr{F}{ }_{t}, \mathscr{P}, \mathscr{W}, \mu\right)$ satisfies the condition (2.29) below 


$$
\mu^{\prime}(t, \cdot)=\delta_{U(t)}(\cdot)
$$

where $U$ is an $\mathscr{F}_{t}$-adapted process with values in $\Gamma$ and $\delta_{x}=\delta$-measure at $x, \mathscr{R}$ is called an admissible system and denoted by $A=(\Omega, \mathscr{F}, \mathscr{F} t, \mathscr{P}, \mathscr{W}, \mathscr{U})$ or $A=(W, U)$. Let us set

$$
a_{N}=\left\{A=(W, U) ; U \text { is } W \text {-adapted and } U(t)=U\left(\left[2^{N} t\right] 2^{-N}\right)\right\}
$$

where [ ] = Gauss symbol. Now using the same arguments as [5. Sect. 5], we obtain the approximation theorem below

TheOREM 2.2 .

$$
\begin{gathered}
V(\phi)=\liminf _{N \rightarrow \infty} E \int_{0}^{\infty} e^{-\lambda t} F(q(t, \phi, A)) d t \\
\inf _{\mathscr{R}} E \int_{0}^{T} e^{-\lambda t} F(q(t, \phi, \mathscr{R})) d t+e^{-\lambda t} \mathrm{G}(q(T, \phi, \mathscr{R})) \\
=\liminf _{N \rightarrow \infty} E \int_{0}^{T} e^{-\lambda t} F(q(t, \phi, A)) d t+e^{-\lambda T} G(q(t, \phi, A))
\end{gathered}
$$

for $G \in \operatorname{BUG}(H)$ (= the space of all bounded and uniformly continuous functions on $H$, with the supremum norm.)

Thus the Bellman principle is proved by the routine.

Theorem 2.3 (Bellman principle). For any $T$

$$
\begin{aligned}
V(\phi) & =\inf _{\mathscr{R}} E \int_{0}^{T} e^{-\lambda t} F(q(t, \phi, \mathscr{R})) d t+e^{-\lambda T} V(q(T, \phi, \mathscr{R})) \\
& =\lim _{N \rightarrow \infty} \inf _{a_{N}} E \int_{0}^{T} e^{-\lambda t} F(q(t, \phi, A)) d t+e^{-\lambda \Gamma} V(q(T, \phi, A))
\end{aligned}
$$

holds.

For the proof we show two inequalities, i.e.

$$
V(\phi) \leqq \liminf _{N \rightarrow \infty} E \int_{0}^{T} e^{-\lambda t} F(q(t, \phi, A)) d t+e^{-\lambda T} V(q(T, \phi, A))
$$

and the converse inequality. The converse is easy by the definition of $V$, but not for (2.32). With this consideration, we will call the following inequality (2.33) a sub Bellman principle

$$
\begin{aligned}
& V(\phi) \leqq E \int_{0}^{T} e^{-\lambda t} F(q(t, \phi, u)) d t+e^{-\lambda T} V(q(T, \phi, u)) \\
& \text { for } T<\infty \text { and } u \in \Gamma .
\end{aligned}
$$

In Section 5 we will use (2.33), (see Theorem 5.2). 


\section{§ 3. Properties of $J$ and $V$}

Hereafter we always assume the following conditions (A.4) and (A.5), besides (A.1)-(A.3).

(A.4) $\lambda>c$, where $c$ is the constant in (2.15).

(A.5) $F$ is the twice Fréchet differentiable and DF and $D^{2} F$ satisfy (3.1) and (3.2) below

$$
\|D F(\phi)\| \leqq \tilde{K}, \quad \llbracket D^{2} F \rrbracket \leqq \tilde{K} \quad \text { for } \phi \in H,
$$

with some constant $\tilde{K}$, and

$$
\llbracket D^{2} F(\phi)-D^{2} F(\psi) \rrbracket \rightarrow 0 \text { uniformly, as }\|\phi-\psi\| \rightarrow 0,
$$

where we identify $D F(\phi)$ with an element of $H$ and $D^{2} F(\phi)$ is identified with a bounded self-adjoint operator on $H . L(H)$ denotes the space of all bounded self-adjoint operators on $H$ and $[\cdot]$ is its norm.

For example, a taim function $F$, written by $F(\phi)=f\left(\left(e_{1}, \phi\right), \cdots,\left(e_{n}, \phi\right)\right)$ with a smooth function $f$ on $R^{n}$, satisfies (A.5).

First we study differentiability of $\bar{J}$. Putting

$$
G(\phi, \mathscr{R} ; \psi)=\int_{0}^{\infty} e^{-\lambda t} E(D F(q(t, \phi, \mathscr{R})), p(t, \psi, \mathscr{R})) d t
$$

we see, from (A.5).

$$
\begin{gathered}
|J(\phi+\varepsilon \psi, \mathscr{R})-J(\phi, \mathscr{R})-\varepsilon G(\phi, \mathscr{R} ; \psi)| \\
=\mid E \int_{0}^{\infty} e^{-\lambda t} F(q(t, \psi+\varepsilon \psi, \mathscr{R}))-F(q(t, \phi, \mathscr{R})) \\
\quad-\varepsilon(D F(q(t, \phi, \mathscr{R}), p(t, \psi, \mathscr{R}))) d t \mid \\
\leqq \frac{\varepsilon^{2}}{2} \int_{0}^{\infty} e^{-\lambda t} \tilde{K} E\|p(t, \psi, \mathscr{R})\|^{2} d t \leqq \frac{\varepsilon^{2} \tilde{K}}{2(\lambda-c)}\|\psi\|^{2} .
\end{gathered}
$$

Moreover we have

$$
\begin{aligned}
& |G(\phi+\xi, \mathscr{R} ; \psi)-G(\phi, \mathscr{R} ; \psi)| \\
& \quad \leqq\left|\int_{0}^{\infty} e^{-\lambda t} E(D F(q(t, \phi+\xi, \mathscr{R}))-D F(q(t, \phi, \mathscr{R})), p(t, \psi, \mathscr{R})) d t\right| \\
& \quad \leqq \int_{0}^{\infty} e^{-\lambda t} \tilde{K} E\|p(t, \xi, \mathscr{R})\|\|p(t, \psi \cdot \mathscr{R})\| d t \\
& \quad \leqq \tilde{K} \int_{0}^{\infty} e^{-\lambda t} \cdot e^{-c t}\|\xi\|\|\psi\| d t=\frac{\tilde{K}}{\lambda-c}\|\xi\|\|\psi\| .
\end{aligned}
$$


On the other hand, $G(\phi, \mathscr{R} ; \psi)$ is linear in $\psi$, since $p(t, \psi, \mathscr{R})$ is a unique solution of linear SPDE (2.19). By (3.5), $G(\cdot, \mathscr{R}, \cdot)$ can be extended on $H \times H$, say $\bar{G}(\cdot, \mathscr{R}, \cdot)$, and $\bar{G}(\phi, \mathscr{R} ; \psi)$ is linear in $\psi \in H$ and satisfies

$$
|\bar{G}(\phi, \Re ; \psi)| \leqq \tilde{K} /\left(\lambda-\frac{c}{2}\right)
$$

and

$$
|\bar{G}(\phi+\xi, \mathscr{R} ; \psi)-\bar{G}(\phi, \mathscr{R} ; \psi)| \leqq \frac{\tilde{K}}{\lambda-c}\|\xi\|\|\psi\|
$$

Again (3.4) derives

$$
|\bar{J}(\phi+\varepsilon \psi, \mathscr{R})-\bar{J}(\phi, \mathscr{R})-\varepsilon \bar{G}(\phi, \mathscr{R} ; \psi)| \leqq \frac{\varepsilon^{2} \tilde{K}}{2(\lambda-c)}\|\psi\|^{2} .
$$

Since $\bar{G}(\phi, \mathscr{R} ; \psi)$ is linear in $\psi,(3.7)$ and (3.8) yield the following proposition.

Proposition 3.1. $D \bar{J}(\phi, \mathscr{R})=\bar{G}(\phi, \mathscr{R} ; \cdot)$, and

$$
\|D \bar{J}(\phi+\xi, \mathscr{R})-D \bar{J}(\phi, \mathscr{R})\| \leqq \frac{\tilde{K}}{\lambda-c}\|\xi\| .
$$

From (3.6), we can easily see

$$
|\bar{V}(\phi)-V(\psi)| \leqq\left(\tilde{K} /\left(\lambda-\frac{c}{2}\right)\right)\|\phi-\psi\| .
$$

For the differentiability of $\bar{V}$, we first show its concavity. A contiunous function $h$ is called semi-concave, if, with some positive $c$

$$
h(\phi+\psi)+h(\phi-\psi)-2 h(\phi) \leqq c\|\psi\|^{2}, \quad \text { for any } \phi, \psi \in \mathfrak{S} .
$$

Now we first show

Proposition 3.2. $\bar{V}$ is semi-concave.

Proof. It is enough to show (3.11) below.

(3.11) $V(\phi+\psi)+V(\phi-\psi)-2 V(\phi) \leqq \frac{\tilde{K}}{\lambda-c}\|\psi\|^{2}, \quad$ for $\phi, \psi \in \mathfrak{F}$.

From (A.5), we see

$$
\begin{aligned}
& J(\phi+\psi, \mathscr{R})+J(\phi-\psi, \mathscr{R})-2 J(\phi, \mathscr{R}) \\
& \quad=E \int_{0}^{\infty} e^{-\lambda t} F(q(t, \phi+\psi, \mathscr{R})+F(q(t, \phi-\psi, \mathscr{R}))-2 F(q(t, \phi, \mathscr{R}))) d t \\
& \quad=E \int_{0}^{\infty} e^{-\lambda t} \tilde{K}\|p(t, \psi, \mathscr{R})\|^{2} d t \leqq \frac{\tilde{K}}{\lambda-c}\|\psi \cdot\|^{2} .
\end{aligned}
$$


Using the inequality "inf $x_{i}+\inf y_{i}-\inf z_{i} \leqq \sup \left(x_{i}+y_{i}-z_{i}\right)$ ", we obtain (3.11).

Let us set

$$
v(\phi)=\bar{V}(\phi)-\frac{\tilde{K}}{2(\lambda-c)}\|\phi\|^{2}, \quad \text { for } \phi \in H .
$$

Then $v$ is locally Lipschitz and concave.

\section{§4. Differentials of $\bar{V}$}

Now we will study differential of $v$ of (3.13), appealing to the arguments of convex analysis. For any $\phi$ and $\psi$ in $H, v(\phi+\theta \psi)-v(\phi)$ has the right and left derivatives with respect to $\theta$, say $\partial^{+} v(\phi ; \psi)$ and $\partial^{-} v(\phi ; \psi)$ respectively, namely

$$
\partial^{+} v(\phi ; \psi)=\lim _{0 \downarrow 0} \frac{v(\phi+\theta \psi)-v(\phi)}{\theta}
$$

and

$$
\partial^{-} v(\phi ; \psi)=\lim _{\theta \uparrow 0} \frac{v(\phi+\theta \psi)-v(\phi)}{\theta}=-\partial^{+} v(\phi ;-\psi) .
$$

Moreover the following properties hold.

$$
\partial^{+} v(\phi ; \psi)=\sup _{\theta>0} \frac{v(\phi+\theta \psi)-v(\phi)}{\theta}
$$

and

$$
\partial^{+} v(\phi ; \psi) \leqq \partial^{-} v(\phi ; \psi)
$$

Using the standard arguments of convex analysis [cf. 6], we have following Propositions 4.1-4.3.

Proposition 4.1.

$$
\begin{aligned}
& \partial^{+} v(\phi ; \psi) \text { is lower semi-continuous } \\
& \left|\partial^{+} v(\phi ; \psi)-\partial^{+} v(\phi ; \eta)\right| \leqq \frac{\tilde{K}}{\lambda-c}(1+\|\phi\|)\|\psi-\eta\| \\
& \varlimsup_{\xi \rightarrow \phi} \partial^{+} v(\xi ; \psi)=\partial^{-} v(\phi ; \psi)
\end{aligned}
$$

Proof. (4.5) is easy by the routine method of convex analysis. Since (4.6) is clear by (3.10) and (3.13), we will only show (4.7). 
Since $v(\phi+\theta \psi)$ is concave in $\theta \in(-\infty, \infty), \partial^{+} v(\phi+\varepsilon \psi ; \psi)$ is decreasing in $\varepsilon$. Moreover

$$
\lim _{\varepsilon \neq 0} \partial^{+} v(\phi+\varepsilon \psi ; \psi) \geqq \partial^{-} v(\phi ; \psi)
$$

Thus we get

$$
\varlimsup_{\xi \rightarrow \theta} \partial^{+} v(\xi ; \psi) \geqq \lim _{\varepsilon \uparrow 0} \partial^{+} v(\phi+\varepsilon \psi ; \psi) \geqq \partial^{-} v(\phi ; \psi) .
$$

On the other hand, we see from (4.2) (4.4) and (4.5),

$$
\begin{aligned}
\varlimsup_{\xi \rightarrow \phi} \partial^{+} v(\xi ; \psi) & \leqq \varlimsup_{\xi \rightarrow \phi} \partial^{-} v(\xi ; \psi)=\varlimsup_{\xi \rightarrow \phi}\left(-\partial^{+} v(\xi ;-\psi)\right) \\
& =-\varliminf_{\xi \rightarrow \phi} \partial^{+} v(\xi ;-\psi) \leqq-\partial^{+}(\phi ;-\psi)=\partial^{-} v(\phi ; \psi) .
\end{aligned}
$$

Combining (4.11) with (4.10), we have (4.7).

Proposition 4.2. $\partial^{+} v(\cdot ; \psi)$ is continuous at $\phi$, if and only if

$$
\partial^{+} v(\phi ; \psi)=\partial^{-} v(\phi ; \psi)
$$

holds, namely two-side derivative, say $\partial v(\phi ; \psi)$, exists.

Proof. Suppose (4.12) holds. Then (4.5) and (4.7) derive

$$
\partial^{+} v(\phi ; \psi) \leqq \lim _{\xi \rightarrow \phi} \partial^{+} v(\xi ; \psi) \leqq \lim _{\xi \rightarrow \phi} \partial^{+} v(\xi ; \psi)=\partial^{-} v(\phi ; \psi) .
$$

Hence $\partial^{+} v(\cdot ; \psi)$ is continuous at $\phi$.

For the converse, we assume

$$
\partial^{+} v(\phi ; \psi)=\varlimsup_{\xi \rightarrow \phi} \partial^{+} v(\xi ; \psi)=\varliminf_{\xi \rightarrow \phi} \partial^{+} v(\xi ; \psi) .
$$

Then (4.7) derives (4.12).

For $\psi \in H$, we will define $\mathbf{D}_{\psi}$ and $h_{\psi}$ by

$$
\mathbf{D}_{\psi}=\{\phi \in H ; \partial v(\phi ; \psi) \text { exists }\}
$$

and

$$
h_{\psi}(\phi)=\partial^{+} v(\phi ; \psi)+\partial^{+} v(\phi ;-\psi) \quad\left(=\partial^{+} v(\phi ; \psi)-\partial^{-} v(\phi ; \psi)\right),
$$

respectively. Then $h_{\psi}$ is lower semi-continuous by (4.5) and $h_{\psi}(\phi)<0$ for $\phi \notin \mathbf{D}_{\psi}$. Let us set

$$
S_{p}=\left\{\phi \in H ; h_{\psi}(\phi) \leqq-\frac{1}{p}\right\}, \quad p=1,2, \cdots .
$$


Then $S_{p}$ is closed and increasing to $\mathbf{D}_{\psi}^{\mathrm{c}}$ as $p \uparrow \infty$.

Proposition 4.3. $\left\{\theta \in(-\infty, \infty) ; \xi+\theta \psi \notin \mathbf{D}_{\psi}\right\}$ is countable, for any $\xi$ and $\psi$ in $H$.

Proof. For the proof we may assume $(\psi, \xi)=0$. Put

$$
g^{+}(\theta)=\partial^{+} v(\xi+\theta \psi ; \psi) \text { and } g^{-}(\theta)=\partial^{-} v(\xi+\theta \psi ; \psi) .
$$

Then $g^{+}$and $g^{-}$are locally bounded and decreasing. Moreover

$$
g^{-}(\theta) \geqq g^{+}(\theta) \geqq \lim _{\varepsilon \downarrow 0} g^{-}(\theta+\varepsilon) \quad\left(\text { say } g^{-}(\theta+)\right)
$$

holds, and $h_{\psi}(\xi+\theta \psi)=g^{+}(\theta)-g^{-}(\theta) \geqq g^{-}(\theta+)-g^{-}(\theta)$.

Thus, for $\delta>0,\left\{\theta \in[\alpha, \beta] ; g^{-}(\theta+)-g^{-}(\theta)<-\delta\right\}$ is finite. This implies $\left\{\theta \in[\alpha, \beta] ; h_{\psi}(\xi+\theta \psi)<-\delta\right\}$ is finite and $\left\{\theta \in(-\infty, \infty) ; \xi+\theta \psi \in S_{p}\right\}$ is countable. Since $\mathbf{D}_{\psi}^{c}=\cup_{p} S_{p}$, we complete the proof.

Let $e_{k}, k=1,2, \cdots$ be an orthonormal base of $H$. Let $X_{k}, k=1,2$, .. be independent $N\left(0, v_{k}\right)$-distributed Gaussian random variables on a probability space $(\Omega, \mathscr{F}, \mathscr{P})$. We assume

$$
v_{k}>0 \text { for } k=1,2, \cdots \text { and } \sum v_{k}<\infty .
$$

Hence $P\left(\sum X_{k}^{2}<\infty\right)=1$ and

$$
P\left(\sum X_{k} e_{k} \in H\right)=1 \text {. }
$$

$Q$ denotes the probability law of $\sum X_{k} e_{k}$. So, $(H, \sigma(H), Q)$ is a probability space and $Y_{k}(w)=\left(w, e_{k}\right) / \sqrt{v_{k}}, k=1,2, \cdots$, turn out to be $N(0,1)$-distributed independent random variables on $(H, \sigma(H), Q)$. For finite sum of $e_{k}$, say $\psi=\sum_{j=1}^{n} r_{j} e_{j}(\neq 0)$, we can take a new orthogonal base $f_{k}, k=$ $1,2, \cdots$ such that $f_{1}=\psi$, linear space of $\left(f_{1} \cdots, f_{n}\right)=$ linear space of $\left(e_{1}, \cdots e_{n}\right)$ and $f_{n+i}=e_{n+i}, i=1,2, \cdots$ and $Z_{k} ; Z_{k}(w)=\left(w, f_{k}\right)$, are independent Gaussian random variables. Appealing to Proposition 4.3, we show

Proposition 4.4. $Q\left(\mathbf{D}_{\psi}^{c}\right)=0$, for $\psi=\sum_{j=1}^{n} r_{j} e_{j}(\neq 0)$.

Proof. Let us set

$$
Y(w)=w-Z_{1}(w) \psi=\sum_{k=2}^{\infty} Z_{k}(w) f_{k}, \quad \text { for } w \in H .
$$

Then $Y$ and $Z_{1} \psi$ are independent. Hence, for any bounded Borel function $g$ on $H \times H$, we have 


$$
E\left(g\left(Y, Z_{1} \psi\right) / \sigma(Y)\right)=\int_{H} g(Y, u) \mu(d u)
$$

where $\mu$ is the probability distribution of $Z_{1} \psi$. Since $Z_{1}$ has a normal distribution $N$, we get

$$
\int_{H} g(Y, u) \mu(d u)=\int_{-\infty}^{\infty} g(Y, \theta \psi) N(d \theta) .
$$

This derives, for any $A \in \sigma(H)$

$$
Q(A)=\int_{H}\left(\int_{-\infty}^{\infty} I_{A}(y+\theta \psi) N(d \theta)\right) \nu(d y)
$$

where $\nu$ is the probability distribution of $Y$. Now putting $A=\mathbf{D}_{\psi}^{c}$, Proposition 4.3 completes the proof.

Let us set

$$
\mathscr{A}=\left\{\sum_{k=1}^{n} r_{k} e_{k} \neq 0 ; r_{k}=\text { rational, } k=1,2, \cdots n, n=1,2, \cdots\right\}
$$

and

$$
\mathbf{D}=\bigcap_{\psi \in \mathscr{\infty}} \mathbf{D}_{\psi} .
$$

Then $\mathscr{A}$ is a countable dense set of $H$ and $\mathbf{D} \in \sigma(H)$. Now Proposition 4.4 yields

Theorem 4.1. For $\phi \in \mathbf{D}, \partial v(\phi ; \psi)$ and $\partial v(\phi ;-\psi)$ exist for $\psi \in H$.

Proof. For $\phi \in \mathbf{D}$ and $\psi \in \mathscr{A},(4.15)$ implies

$$
\partial^{+} v(\phi ; \psi)=-\partial^{+} v(\phi ;-\psi) .
$$

But, since $\mathscr{A}$ is dense, (4.22) still holds for $\psi \in H$ by (4.6). This completes the proof.

Next we will give one remark on a full probability set of $H$.

Proposition 4.5. If $Q(A)=1$ for a set $A$ of $H$, then $A$ is dense in $H$.

Proof. Let us set $X_{k}(w)=\left(w, e_{k}\right), k=1,2, \ldots$. Then $X_{k}$ is independent and $N\left(0, v_{k}\right)$-distributed. From (A.6), we can choose, for $\varepsilon>0$, $N(\varepsilon)$ such that $N>n$ and

$$
Q\left(\sum_{k=N+1}^{\infty} X_{k}^{2}<\varepsilon\right)>\frac{1}{2}
$$


holds. On the other hand

$$
Q\left(\sum_{k=1}^{N}\left|X_{k}-r_{k}\right|^{2}<\varepsilon\right)>0
$$

where $r_{k}=0$ for $k \geqq n+1$, since $X_{1}, \cdots, X_{n}$ are independent Gaussian random variables with $E X_{k}^{2}>0$. Moreover the independence of $X_{k}$ implies

$$
Q(B(2 \varepsilon: \psi)) \geqq Q\left(\sum_{k=1}^{N}\left|X_{k}-r_{k}\right|^{2}<\varepsilon\right) Q\left(\sum_{k=N+1}^{\infty} X_{k}^{2}<\varepsilon\right)>0
$$

where $B(c ; \psi)=\left\{w \in H ;\|w-\psi\|^{2}<c\right\}$. Therefore

$$
Q(A \cap B(2 \varepsilon ; \psi))>0 \text {. }
$$

Thus, for any $\psi \in \mathscr{A}$ and $\varepsilon>0, A \cap B(2 \varepsilon: \psi)$ is non-empty, This completes the proof.

Next we will show that $\partial v(\phi ; \psi)$ is linear in $\psi$. From the definition, $\partial^{+} v(\phi, \psi)$ is clearly positively homogeneous in $\psi$, i.e. for $\phi, \xi \in H$ and $\theta>0$,

$$
\theta \partial^{+} v(\phi ; \xi)=\partial^{+} v(\phi ; \theta \xi) \text { and } \theta \partial^{-} v(\phi ; \xi)=\partial^{-} v(\phi ; \theta \xi) \text {. }
$$

Therefore, for $\phi \in \mathbf{D}, \xi \in H$ and $\theta>0$, we see

$$
\partial^{+} v(\phi ;-\theta \xi)=-\partial^{-} v(\phi ; \theta \xi)=-\theta \partial^{-} v(\phi ; \xi)=-\theta \partial^{+} v(\phi ; \xi) .
$$

For $\phi, \xi, \eta \in H$, we will prove inequalities (4.28) and (4.29) below

$$
\begin{aligned}
& \partial^{+} v(\phi ; \xi+\eta) \leqq \partial^{-} v(\phi ; \xi)+\partial^{+} v(\phi ; \eta) \\
& \partial^{-} v(\phi ; \xi+\eta) \leqq \partial^{+} v(\phi ; \xi)+\partial^{-} v(\phi ; \eta)
\end{aligned}
$$

Proof. For $\varepsilon>0$, we have

$$
\begin{aligned}
\frac{v(\phi+\varepsilon \xi+\varepsilon \eta)-v(\phi+\varepsilon \eta)}{\varepsilon} & \leqq \sup _{\theta>0} \frac{v(\phi+\varepsilon \xi+\theta \eta)-v(\phi+\varepsilon \eta)}{\theta} \\
& =\partial^{+} v(\phi+\varepsilon \eta ; \xi) .
\end{aligned}
$$

Hence we see

$$
\frac{v(\phi+\varepsilon \xi+\varepsilon \eta)-v(\phi)}{\varepsilon} \leqq \partial^{+} v(\phi+\varepsilon \eta ; \xi)+\frac{v(\phi+\varepsilon \eta)-v(\phi)}{\varepsilon} .
$$

As $\varepsilon \downarrow 0$, we get (4.28) by virtue of (4.7).

Appealing to " $\partial^{-} v(\phi ; \psi)=-\partial^{+} v(\phi ;-\psi) "$, (4.28) yields (4.29).

Now Theorem 4.1 derives, for $\phi \in \mathbf{D}$

$$
\partial v(\phi ; \xi+\eta)=\partial v(\phi ; \xi)+\partial v(\phi ; \eta), \quad \text { for } \xi, \eta \in H .
$$


Combining (4.30) with (4.26) and (4.27), we have

$$
\partial v(\phi ; a \xi+b \eta)=a v(\phi ; \xi)+b v(\phi ; \eta), \quad \text { for } \phi \in \mathbf{D} .
$$

Again (4.6) implies " $\partial v(\phi ; \cdot) \in H^{*}$ ". Since $\|\phi\|^{2}$ is differentiable, we get

Theorem 4.2. $\bar{V}$ is Gâteux differentiable at $\phi \in \mathbf{D}$ and its Gâteux derivative $\partial \bar{V}(\phi) \in H^{*}$.

Let $\overline{\mathscr{R}}=\overline{\mathscr{R}}(\phi)$ be an optimal one, which satisfies (2.28). Recalling Proposition 3.1, we have

THEOREM 4.3. $\partial \bar{V}(\phi)=D \bar{J}(\phi ; \overline{\mathscr{R}})$, for $\phi \in \mathbf{D}$.

Proof. Since $\bar{V}(\phi)=\bar{J}(\phi ; \overline{\mathscr{R}})$ and $\bar{V}(\phi+\xi) \leqq \bar{J}(\phi+\xi ; \overline{\mathscr{R}})$ for $\xi \in H$, we see from (3.4)

$$
\begin{aligned}
\bar{V}(\phi+\varepsilon \psi)-\bar{V}(\phi) & \leqq \bar{J}(\phi+\varepsilon \psi ; \overline{\mathscr{R}})-\bar{J}(\phi ; \overline{\mathscr{R}}) \\
& \leqq \varepsilon(D \bar{J}(\phi ; \overline{\mathscr{R}}), \psi)+\frac{\varepsilon^{2} \tilde{K}}{2(\lambda-c)}\|\psi\|^{2} .
\end{aligned}
$$

Dividing both sides by $\varepsilon>0$ and tending $\varepsilon$ to 0 , we get

$$
(\partial \bar{V}(\phi), \psi) \leqq(D \bar{J}(\phi ; \overline{\mathscr{R}}), \psi), \quad \text { for } \psi \in H .
$$

Since $\psi$ is arbitrary in $H$, " $(\partial \bar{V}(\phi),-\psi) \leqq(D \bar{J}(\phi ; \mathscr{R}),-\psi)$ " also holds. This derives

$$
(\partial \bar{V}(\phi), \psi)=(D \bar{J}(\phi ; \overline{\mathscr{R}}), \psi) \quad \text { for } \psi \in H .
$$

Now we complete the proof.

To study the value function $V$, we are concerned with $\mathbf{D} \cap \mathfrak{S}_{\mathrm{g}}$. Taking Hermite base and suitable $v_{k}$, we can prove that $\mathbf{D} \cap \mathfrak{S}$ is dense in $H$. Putting

$$
h_{n}(x)=(-1)^{n} \sqrt{n !^{-1}} \exp \left(\frac{x^{2}}{2}\right)\left(\frac{d}{d x}\right)^{n}\left(\exp \left(\frac{-x^{2}}{2}\right)\right), \quad \text { for } x \in \mathbf{R}^{1},
$$

the Hermite base $e_{\underline{k}}$ is defined as follows.

$$
e_{\underline{k}}(\underline{x})=\prod_{i=1}^{d} h_{k_{i}}\left(x_{i}\right) \exp \left(\frac{-x_{i}^{2}}{2}\right)
$$

for $\underline{x}=\left(x_{1}, \cdots x_{d}\right) \in \mathbf{R}^{d}$ and multi index $\underline{k}=\left(k_{1}, \cdots k_{d}\right), k_{i} \geqq 0$. Using the following properties of $h_{n}$

$$
h_{n}^{\prime}=\sqrt{n} h_{n-1}, \quad h_{n}^{\prime}-x h_{n}=-\sqrt{n+1} h_{n+1} .
$$


we get

$$
x^{2} h_{n}=\sqrt{n(n-1)} h_{n-2}+(2 n+1) h_{n}+\sqrt{(n+1)(n+2)} h_{n+2} .
$$

Therefore we have

$$
\frac{\partial e_{\underline{k}}}{\partial x_{i}}=\frac{1}{2}\left(\sqrt{k_{i}} e_{\underline{\underline{k}}-I_{i}}-\sqrt{k_{i}+1} e_{\underline{\underline{k}}+I_{i}}\right)
$$

and

$$
|\underline{x}|^{2} e_{\underline{\underline{k}}}=\sum_{j=1}^{d} \sqrt{k_{j}\left(k_{j}-1\right)} e_{\underline{\underline{k}-2 I_{j}}}+\left(2 k_{j}+1\right) e_{\underline{\underline{k}}}+\sqrt{\left(k_{j}+1\right)\left(k_{j}+2\right)} e_{\underline{\underline{k}}+2 I_{j}}
$$

where $I_{j}$ is the $j$ th unit vector $(0, \cdots 0,1,0, \cdots 0)$ and $\underline{k}+\underline{p}=\left(k_{1}+p_{1}\right.$, $\left.\cdots k_{d}+p_{d}\right)$.

For $\phi=\sum c_{\underline{k}} e_{\underline{\underline{k}}}$, (4.36) yields

$$
\frac{\partial \phi}{\partial x_{i}}=\frac{1}{2}\left(\sum c_{\underline{\underline{k}}} \sqrt{k_{i}} e_{\underline{\underline{k}-I_{i}}}-c_{\underline{\underline{k}}} \sqrt{k_{i}+1} e_{\underline{\underline{k}}+I_{i}}\right) .
$$

So, $\partial \phi / \partial x_{i} \in H$, if and only if $\sum c_{\underline{\underline{k}}}^{2} k_{i}<\infty$. Repeating this argument, we have

Proposition 4.6. If $\sum c_{\underline{k}}^{2}|\underline{k}|^{p}<\infty$, then $\phi \in H^{p}$.

On the other hand, (4.37) implies that, if $\sum c_{\underline{k}}^{2}|\underline{k}|^{2}<\infty$, then $\left(1+|\underline{x}|^{2}\right) \phi$ $\in H$. Hence $\left(1+|\underline{x}|^{2}\right)\left(\partial \phi / \partial x_{i}\right) \in H$, if $\sum c_{\underline{k}}^{2}|\underline{k}|^{3}<\infty$. Therefore, if $\sum\left(\phi, e_{\underline{k}}\right)^{2}|\underline{k}|^{4}$ $<\infty$, then $\phi \in \mathfrak{S}$.

Putting

$$
v_{\underline{k}}=|\underline{k}|^{-d-5}
$$

we will define a probability $Q$ on $H$ in the same way as (4.18) and obtain a full probability set $\mathbf{D}$ of (4.21). Since the number of elements of $\{\underline{k} ;|\underline{k}|=n\}=\left(\begin{array}{c}n+d-1 \\ d-1\end{array}\right)=O\left(n^{d-1}\right), " ~ \sum v_{k}|\underline{k}|^{4}<\infty "$ holds. Therefore $Q(\mathfrak{C})=1$. Hence we get

$$
Q(\mathbf{D} \cap \mathfrak{S})=1
$$

Moreover Theorem 4.3 derives that, for $\phi \in \mathfrak{S} \cap \mathbf{D}$,

$$
\begin{aligned}
(\partial \bar{V}(\phi), \psi) & =\lim _{\varepsilon \rightarrow 0} \frac{1}{\varepsilon}(V(\phi+\varepsilon \psi)-V(\phi)) \\
& \left.=\lim _{\varepsilon \rightarrow 0} \frac{1}{\varepsilon}\left(J\left(\phi+\varepsilon \psi ; \mathscr{R}^{*}\right)-J\left(\phi ; \mathscr{R}^{*}\right)\right)=\partial \bar{J}\left(\phi ; \mathscr{R}^{*}\right), \psi\right)
\end{aligned}
$$

holds, for any $\psi \in \mathfrak{S}$, where $\mathscr{R}^{*}=\mathscr{R}^{*}(\phi)$ is an optimal relaxed system. 


\section{§ 5. Subsolution of Bellman equation}

Suppose $\bar{V}$ is smooth, namely satisfies the condition (A.5). Then Ito's formula derives, for $\phi \in \mathfrak{S}$ and an admissible system $A=(W, U)$,

$$
\begin{aligned}
E e^{-\lambda t} V(q(t))-V(\phi)= & \int_{0}^{t} e^{-\lambda s} E(-\lambda V(q(s))+(D V(q(s)), L(U(s)) q(s)) \\
& +\frac{1}{2}\left(D^{2} V(q(s)) M q(s), M q(s)\right) d s
\end{aligned}
$$

where $q=q(\cdot, \cdot, A)$. Appealing to (2.16), the Bellman principle show that $\bar{V}$ satisfies the Bellman equation (4.2) below.

Proposition 5.1. If $\bar{V}$ satisfies (A.5), then

$$
\begin{array}{r}
0=\sup _{u \in \Gamma}\left(-\frac{1}{2}\left(D^{2} \bar{V}(\phi) M \phi, M \phi\right)-(D \bar{V}(\phi), L(u) \phi)+\lambda \bar{V}(\phi)-F(\phi)\right) \\
\text { for } \phi \in \mathfrak{S} .
\end{array}
$$

Proof. First we evaluate the integrand of (5.1), for small $t$. Here $K_{i}$ denotes a constant independent of $A, \phi$ and $s$.

$$
\begin{aligned}
& E|(D \bar{V}(q(s))-D \bar{V}(\phi), L(U(s)) q(s))| \\
& \quad \leqq K_{2} E\|q(s)-\phi\|\|q(s)\|_{2} \leqq K_{2} \sqrt{s} \sqrt{\|\phi\|_{1}^{2}+A_{1}} \sqrt{\|\phi\|_{2}^{2}+A_{2}} \\
& \leqq K_{3} \sqrt{s}\left(\|\phi\|_{2}^{2}+1\right) \\
& E|(D \bar{V}(\phi), L(U(s)) q(s)-L(U(s)) \phi)| \leqq K_{4} E\|q(s)-\phi\|_{2} \\
& \quad \leqq K_{5} \sqrt{s} \sqrt{\|\phi\|_{3}^{2}+1}
\end{aligned}
$$

From (5.3) and (5.4), we see

$$
E|(D \bar{V}(s), L(U(s)) q(s))-(D \bar{V}(\phi), L(U(s)) \phi)| \leqq K_{6} \sqrt{s}\left(\|\phi\|_{3}+\|\phi\|_{2}^{2}+1\right)
$$

Repeating the similar arguments, we have

$$
E \mid\left(D^{2} \bar{V}(q(s)) M q(s), M q(s)\right)-\left(D^{2} \bar{V}(q(s)) M \phi, M \phi \mid \leqq K_{7} \sqrt{s}\left(\|\phi\|_{2}^{2}+1\right) .\right.
$$

For $\varepsilon>0$, there is $\delta=\delta(\varepsilon)>0$, such that

$$
\llbracket D^{2} \bar{V}(\xi)-D^{2} \bar{V}(\eta) \rrbracket<\varepsilon, \quad \text { whenever }\|\xi-\eta\|<\delta,
$$

by virtue of (A.5). Hence we have

$$
\begin{aligned}
E\left(\left(D^{2} \bar{V}(q(s))-L^{2} \bar{V}(\phi)\right) M \phi, M \phi\right) \mid & \leqq K_{8}\|M \phi\|^{2}\left(\varepsilon+\frac{E\|q(s)-\phi\|^{2}}{\delta^{2}}\right) \\
& \leqq K_{9}\|M \phi\|^{2}\left(\varepsilon+\sqrt{s} \frac{\|\phi\|_{1}^{2}+1}{\delta^{2}}\right)
\end{aligned}
$$


Combining (5.6) with (5.8), we have

$$
\begin{aligned}
& \left|E\left(D^{2} \bar{V}(q(s)) M q(s), M q(s)\right)-\left(D^{2} \bar{V}(\phi) M \phi, M \phi\right)\right| \\
& \quad \leqq K_{10}\left(\sqrt{s}\|\phi\|_{2}^{2}+1\right)+\left(\|\phi\|_{1}^{2}+1\right)\left(\varepsilon+\frac{\|\phi\|_{1}^{2}+1}{\delta^{2}} \sqrt{s}\right) .
\end{aligned}
$$

Now recalling (5.1), we get

$$
\begin{aligned}
& E e^{-\lambda t} V(q(t))-V(\phi) \\
& \quad=E \int_{0}^{t} \frac{1}{2}\left(D^{2} \bar{V}(\phi) M \phi, M \phi\right)+(D \bar{V}(\phi), L(U s) \phi)-\lambda V(\phi) d s+o(t) .
\end{aligned}
$$

where $o(t) / t \rightarrow 0$, uniformly in $A$, as $t \rightarrow 0$.

On the other hand, the Bellman principle yields

$$
0=\inf _{A} E \frac{1}{t}\left[\int_{0}^{t} e^{-\lambda s} F(q(s, \phi, A)) d s+e^{-\lambda t} V(q(t, \phi, A))-V(\phi)\right] .
$$

Combining (5.10) with (5.11), we have

$$
\begin{array}{r}
0=\inf _{A}\left(F(\phi)-\lambda V(\phi)+\frac{1}{2}\left(D^{2} \bar{V}(\phi) M \phi, M \phi\right)\right. \\
+\frac{1}{t} \int_{0}^{t} E(D \bar{V}(\phi), L(U(s)) \phi) d s+o(1) .
\end{array}
$$

Since $(D \bar{V}(\phi), L(U(s)) \phi) \geqq \inf _{u \in L}(D \bar{V}(\phi), L(u) \phi)$, we see

$$
\begin{aligned}
& \inf _{A} \int_{0}^{t} E(D \bar{V}(\phi), L(U(s)) \phi) d s \geqq t \inf _{u \in \Gamma}(D \bar{V}(\phi), L(u) \phi) \\
& \quad=\inf _{u \in \Gamma} t(D \bar{V}(\phi), L(u) \phi)=\inf _{u \in \Gamma} \int_{0}^{t} E(D \bar{V}(\phi), L(u) \phi) d s \\
& \quad \geqq \inf _{A} \int_{0}^{t} E(D \bar{V}(\phi), L(U(s)) \phi) d s .
\end{aligned}
$$

Hence

$$
\inf _{A} \int_{0}^{t} E\left(D \bar{V}(\phi), L(U(s) \phi) d s=t \inf _{u \in \Gamma}(D \bar{V}(\phi), L(u) \phi)\right.
$$

holds. (5.12) and (5.13) complete the proof.

We will give a simple example of smooth $\bar{V}$.

ExAmple. $\Gamma$ denotes the set of all Borel functions on $\mathbf{R}^{d}$ with values in $[-1,1]$ and 0 outside the unit ball $\{|x| \leqq 1\}$. Hence $\Gamma\left(\subset L^{2}\left(\mathbf{R}^{d}\right)\right)$ is a complete separable metric space carring the weak topology. For $e_{1}, \cdots, e_{k}$ 
$\in H$ and $h \in C_{0}^{\infty}\left(\mathbf{R}^{k}\right)$, we will put $c(u)=h\left(\left(u, e_{1}\right), \cdots,\left(u, e_{k}\right)\right)$. Then $c$ is continuous on $\Gamma$. Let us assume

$$
a^{i j}(x, u)=a^{i j}(x), \quad \text { for }(i, j) \neq(0,0), \quad a^{00}(x, u)=c(u)
$$

and $f^{i}(x, u)=0$, for $i=0,1, \cdots d$.

Now we consider the following SPDEs for a relaxed system $(W, \mu)$.

$$
\begin{aligned}
& d q(t)=A q(t) d t+\tilde{c}(t, \mu) d t+M q(t) d W(t), \quad q(0)=\phi \\
& d p(t)=A p(t) d t+M p(t) d W(t), \quad p(0)=\phi
\end{aligned}
$$

where $A=\sum_{(i j) \neq(00) i} \frac{\partial}{\partial x_{i}}\left(a^{i j}(x)\left(\frac{\partial}{\partial x_{j}}\right)\right)$. Since $c$ does not depend on $x$, Ito's formula yields

$$
q(t)=\exp \left(\int_{0}^{t} \tilde{c}(s, \mu) d s\right) p(t) .
$$

On the other hand, there exist $u^{*}$ such that $\min _{u \in \Gamma} c(u)=c\left(u^{*}\right)$. So $\tilde{c}(s, \mu) \geqq c\left(u^{*}\right)$, and $\|q(t)\| \geqq \exp \left(c\left(u^{*}\right) t\right)\|p(t)\|$. If the function $F$ has the form $F(\psi)=f\left(\|\psi\|^{2}\right)$ with smooth and non-increasing $f$, then $\mu^{\prime}(t, \cdot)=\delta_{u^{*}}(\cdot)$ is optimal for any $\phi$. Therefore using the same arguments as Proposition 3.1, we obtain the smoothness of $\bar{V}$ by the smooth condition of $f$.

We will study the connection between $\bar{V}$ and a viscosity solution of (5.2), when $\bar{V}$ is not smooth. Let us define $G: L(H) \times H \times \mathbf{R}^{1} \times H^{2} \times \Gamma$ $\rightarrow \mathbf{R}^{1}$, by

$$
G(S, p, a, \phi, u)=-\frac{1}{2}(S M \phi, M \phi)-(p, L(u) \phi)+\lambda a-F(\phi) .
$$

Then the Bellman equation is denoted by

$$
\sup _{u \in \Gamma} G\left(D^{2} v(\phi), D v(\phi), v(\phi), \phi, u\right)=0 .
$$

For $v \in C\left(H, \mathbf{R}^{1}\right)$, the superdifferential at $\phi$, say $D^{+} v(\phi)$, (resp. subdifferential at $\left.\phi, D^{-} v(\phi)\right)$ is defined by

$$
\begin{aligned}
D^{+} v(\phi) & =\left\{(S, p) \in L(H) \times H ; \varlimsup_{\xi \rightarrow 0} \frac{v(\phi+\xi)-v(\phi)-(p, \xi)-\frac{1}{2}(S \xi, \xi)}{\|\xi\|^{2}} \leqq 0\right\} \\
(\text { resp. } & D^{-} v(\phi) \\
= & \left.\left\{(S, p) \in L(H) \times H ; \varliminf_{\xi \rightarrow 0} \frac{v(\phi+\xi)-v(\phi)-(p, \xi)-\frac{1}{2}(S \xi, \xi)}{\|\xi\|^{2}} \geqq 0\right\}\right)
\end{aligned}
$$

Definition 5.1. $v \in \mathrm{BUC}(H)$ is called a subsolution of (5.15), if 


$$
\left\{\phi \in \mathfrak{S} ; D^{+} v(\phi) \text { is not empty }\right\} \text { is dense in } H
$$

and, for any $(S, p) \in D^{+} v(\phi)\left(\phi \in H^{2}\right)$,

$$
\sup _{u \in \Gamma} G(s, p, v(\phi), \phi, u) \leqq 0
$$

holds.

Definition 5.2. $v \in \mathrm{BUG}(H)$ is called a supersolution of (5.15), if

$$
\left\{\phi \in \mathfrak{E} ; D^{-} v(\phi) \text { is not empty }\right\} \text { is dense in } H
$$

and, for any $(S, p) \in D^{-}(\phi)\left(\phi \in H^{2}\right)$,

$$
\sup _{u \in \Gamma} G(S, p, v(\phi), \phi, u) \geqq 0
$$

holds.

If $v$ is both sub and super solution, $v$ is a viscosity solution.

Let us denote by $Z$ the space of all function with sub Bellman principle, namely

$$
\begin{array}{r}
Z=\left\{v \in \operatorname{BUC}(H) ; v(\phi) \leqq E \int_{0}^{t} e^{-\lambda s} F(q(s, \phi, u)) d s+e^{-\lambda t} v(q(t, \phi, u)),\right. \\
\quad \text { for any } \phi \in \mathfrak{S}, t \geqq 0 \text { and } u \in \Gamma\} .
\end{array}
$$

Then $Z$ is closed and convex and $\bar{V} \in Z$ by the Bellman principle.

Proposition 5.2. If $v \in Z$ and satisfies (5.16), then $v$ is a subsolution of (5.15).

Proof. For $(S, p) \in D^{+} v(\phi)$, we can take a smooth function $h$ on $H$, such that

$$
h(\phi)=v(\phi), \quad D h(\phi)=p, \quad D^{2} h(\phi)=S \text { and } h \geqq v \text { on } H .
$$

Moreover there is a constant $b$ such that, for ahy $\psi \in H$,

$$
\begin{aligned}
& |h(\psi)| \leqq b\left(1+\|\psi\|^{2}\right), \quad\|D h(\psi)\| \leqq b(1+\|\psi\|), \\
& \llbracket D^{2} h(\psi) \rrbracket \leqq b \text { and } \lim _{\xi \rightarrow \psi} \llbracket D^{2} h(\xi)-D^{2} h(\psi) \rrbracket=0 .
\end{aligned}
$$

For instance, we will define $h$ according to [1]. Assuming $\phi=0$, we set

$$
w(\psi)= \begin{cases}{\left[v(\psi)-v(0)-(p, \psi)-\frac{1}{2}(S \psi, \psi)\right]^{+} /\|\psi\|^{2}} & \text { for } \psi \neq 0 \\ 0 & \text { for } \psi=0,\end{cases}
$$

where $[a]^{+}=\max (a, 0)$. Then $w$ is continuous at 0 , since $(S, p) \in D^{+} v(\phi)$. 
Let $\rho$ be a smooth increasing function on $[0, \infty)$, such that

$$
\rho(\theta)= \begin{cases}\theta^{3} & \text { on }[0,1) \\ \theta^{2} & \text { on }[2, \infty) .\end{cases}
$$

Put $k(r)=\sup \left\{w(\psi) ;\|\psi\| \leqq r\right.$ and $f(t)=2 \int_{0}^{2 t} \int_{0}^{s} k(r) d r d s+\rho(t)$. Then $f \epsilon$ $C^{2}[0, \infty)$. Hence, $g$, defined by $g(\psi)=f(\|\psi\|)$, satisfies $g(0)=0, D g(0)=0$ and $D^{2} g(0)=0$. Thus $h(\psi)=g(\psi)+v(0)+(p, \psi)+\frac{1}{2}(S \psi, \psi)$ is a required one.

From the definition of $Z$, we see

$$
\begin{aligned}
0 & \leqq E \int_{0}^{t} e^{-\lambda s} F(q(s, \phi, u)) d s+e^{-\lambda t} v(q(t, \phi, u))-v(\phi) \\
& \leqq E \int_{0}^{t} e^{-\lambda s} F(q(s, \phi, u)) d s+e^{-\lambda t} h(q(t, \phi, u))-h(\phi) \\
& \leqq E \int_{0}^{t} e^{-\lambda s}\left(-G\left(D^{2} h(q(s, \phi, u)), D h(q(s, \phi, u)), h(q(s, \phi, u)),\right.\right. \\
& q(s, \phi, u), u)) d s .
\end{aligned}
$$

Dividing both side by $t$ and letting $t$ tend to 0 , we get

$$
0 \geqq G(S, p, v(\phi), \phi, u),
$$

in the same way as Proposition 5.1. Since $u \in \Gamma$ is arbitrary, we complete the proof.

Now we will prove

THEOREM 5.1. $\bar{V}$ is a subsolution.

Proof. Since $\bar{V} \in Z$, we will only show (5.16). Using concavity of $v$ of (3.13), Theorem 4.2 implies

$$
v(\phi+\psi)-v(\phi)-(\partial v(\phi), \psi)-\frac{1}{2}(0 \psi, \psi) \leqq 0, \text { for } \phi \in \mathbf{D}, \psi \in H
$$

This derives

$$
\left(\frac{\tilde{K}}{\lambda-c} I, \partial \bar{V}(\partial)\right) \in D^{+} \bar{V}(\phi), \quad \text { for } \phi \in \mathbf{D},
$$

where $I=$ identity on $H$. Since $\mathbf{D} \cap \mathcal{S}$ is dense, (5.16) holds.

Next we will show the maximum property.

THeorem 5.2. $\bar{V}$ is the maximum subsolution with sub Bellman principle. 
Proof. First we remark that $v \in Z$ satisfies (5.24) below.

$$
v(\phi) \leqq E \int_{0}^{t} e^{-\lambda s} F(q(s, \phi, A)) d s+e^{-\lambda t} v(q(t, \phi, A)),
$$

for $A=(W, U) \in a_{N}$. Put $\Delta=2^{-N}$. For $s \in[k \Delta, k \Delta+\Delta), U(s)$ can be regarded as a constant $(\in \Gamma)$, under the conditional probability $P\left(\cdot / \sigma_{k \Delta}(W)\right.$ ), and $q(s, \phi, U)=q(s-k \Delta, q(k \Delta, \phi, U), U(k \Delta))$ holds. This argument yields

$$
\begin{aligned}
& \left.E \int_{k \Delta}^{t} e^{-\lambda s} F(q(s, \phi, A)) d s+e^{-\lambda t} v(q(t, \phi, A)) / \sigma_{k \Delta}(W)\right) \\
& \quad \geqq e^{-\lambda k \Delta} v(q(k \Delta, \phi, A)) .
\end{aligned}
$$

Repeating this evaluation, we can easily obtain (5.24) by (2.12):

As $t \rightarrow \infty$, (5.24) derives

$$
v(\phi) \leqq \liminf _{N \rightarrow \infty} E \int_{a_{N}}^{\infty} e^{-\lambda s} F(q(s, \phi, A)) d s, \quad \text { for } \phi \in \mathfrak{S} .
$$

Thus (2.30) yields " $v(\phi) \leqq V(\phi)$ for $\phi \in \mathfrak{S C}$ ". Since $\mathfrak{S}$ is dense, we can complete the proof.

The proposition 5.3 below is a partial converse of Proposition 5.2.

Proposition 5.3. Let $\nu$ be a twice differentiable. If $\nu$ is a subsolution, then $\nu \in Z$.

Proof. Since $\left(D^{2} \nu(\psi), D \nu(\psi)\right) \in D^{+} \nu(\psi)$ for any $\psi \in H$ and $q(\cdot, \phi, u) \in$ $C\left([0, \infty), H^{3}\right)$ with probability 1 for $\phi \in \mathfrak{S}$, we see

$$
G\left(D^{2} \nu(q(s, \phi, u)), D \nu(q(s, \phi, u)), \nu(q(s, \phi, u)), q(s, \phi, u), u\right) \leqq 0 .
$$

Hence Ito's formula concludes $\nu \in Z$.

In the customary version of admissible control for SPDE, we use $\Gamma$-valued adapted process, where $\Gamma \subset\left\{u ; \mathbf{R}^{d} \rightarrow \mathbf{R}^{k}\right.$, Borel measurable $\}$. In some cases $\Gamma$ admits only constant functions, for instance, this control is appropriate for the Zakai equation.

\section{§6. Remarks on deterministic control}

When $M=0$, our stochastic control turns out to be a deterministic control for PDE. In this case we usually take $\Gamma$-valued Borel function on $[0, \infty)$ as an admissible control. For an admissible control $u(\cdot)$, we consider PDE

$$
\frac{\partial q}{\partial t}=L(u(t)) q(t), \quad \text { with } q(0)=\phi(\in \mathfrak{S}) .
$$


The value function $w(\phi)$ is defined by

$$
w(\phi)=\inf _{u(\cdot)} \int_{0}^{\infty} e^{-\lambda t} F(q(t, \phi, u(\cdot)) d t .
$$

So this control can be studied in two ways of viewing, either as a stochastic control with $M=0$, or as a deterministic control. First we remark that both value functions coincide.

Proposition 6.1. $w(\phi)=V(\phi)$ for $\phi \in \mathfrak{S}$.

Proof. Since an admissible control $u(\cdot)$ can be regarded as a relaxed system, clearly

$$
V(\phi) \leqq w(\phi)
$$

On the other hand, for $A(W, U) \in a_{N}, U$ turns out to be a $\Gamma$-valued Borel function under the conditional probability $P(\cdot / \sigma(W))$. Hence with probability 1 ,

$$
E\left(\int_{0}^{\infty} e^{-\lambda t} F(q(t, \phi, A)) d t / \sigma(W)\right) \geqq w(\phi)
$$

holds. Now (2.30) completes the proof.

In the same way we can easily see the Bellman principle

$$
w(\phi)=\inf _{u(\cdot)} \int_{0}^{T} e^{-\lambda t} F\left(q(t, \phi, u(\cdot)) d t+e^{-\lambda T} w(q(T, \phi, u(\cdot)) .\right.
$$

When $M=0$, the Bellman equation (5.15) turns out to be the first order equation (6.6) below.

$$
\sup _{u \in \Gamma}(-(D v(\phi), L(u) \phi)+\lambda v(\phi)-F(\phi))=0 .
$$

We will give the definition of viscosity subsolution of (6.6) from the point of first order equation [1].

$$
D_{1}^{+} v(\phi)=\left\{p \in H ; \varlimsup_{\xi \rightarrow 0} \frac{v(\phi+\xi)-v(\phi)-(p, \xi)}{\|\xi\|} \leqq 0\right\}
$$

is called superdifferential.

Definition 6.1. $v \in \mathrm{BUC}(H)$ is called a subsolution of (6.6), if

$$
\left\{\phi \in \mathfrak{S} ; D_{1}^{+} v(\phi) \text { is not empty }\right\} \text { is dense in } H
$$

and, for any $p \in D_{1}^{+} v(\phi),\left(\phi \in H^{2}\right)$, 


$$
\sup _{u \Psi \Gamma}(-(p, L(u) \phi)+\lambda v(\phi)-F(\phi)) \leqq 0
$$

holds.

If $v$ is a subsolution of Definition 5.1, then $v$ turns out to be a subsolution of Definition 6.1, because $p \in D_{1}^{+} v(\phi)$ whenever $(S, p) \in D^{+} v(\phi)$. Hence, Theorem 5.1 concludes that $\bar{V}$ is a subsolution of (6.6).

\section{REFERENCES}

[1] M. G. Crandall and P. L. Lions, Hamilton-Jacobi equations infinite dimensions, Part 1. J. Funct. Anal., 62 (1985), 379-396. Part 2, 65 (1986), 368-405. Part 3, 68 (1986), 214-247. Part 4, 90 (1990), 273-283.

[2] N. Ikeda and S. Watanabe, Stochastic Differential Equations and Diffusion Processes, Kodansha/North-Holland, Tokyo/Amsterdam, 1981.

[ 3 ] N. V. Krylov and B. L. Rozovskii, On characteristics of the degenerate parabolic Ito equations of the second orders, Petrovskii Seminar, 8 (1982), 153-168 (in Russian).

[4] P. L. Lions, Viscosity solutions and optimal stochastic control in infinite dimensions, Part 1, Acta Math. 161 (1988), 243-278. Part 2, Lecture Notes in Math. 1390 (1988), 147-170. Part 3, J. Funct. Anal. 86 (1989), 1-18.

[5] N. Nagase and M. Nisio, Optimal controls for stochastic partial differential equations, SIAM, J. Control Optim., 28 (1990), 186-213.

[6] R. T. Rochafellar, Convex analysis, Princeton Univ. Press, 1972.

[ 7 ] B. L. Rozovskii, Stochastic Evolution Systems, preprint.

Department of Mathematics

Kobe University

Rokko, Kobe 65\%

Japan 